
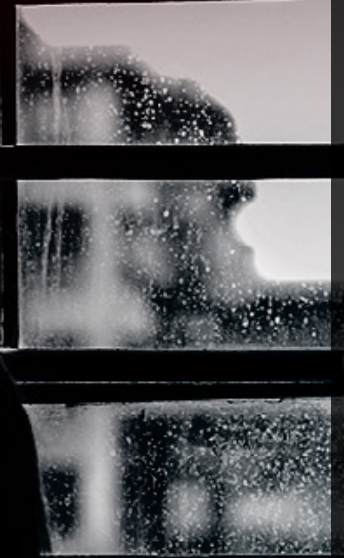

En poco más de una década, México ha sido escenario de diversas expresiones de violencia; entre los delitos más graves se encuentra la desaparición de personas. Más de 61 mil familias han sido afectadas por este fenómeno, lo cual las ha posicionado - sobre todo a las mujeres-, en escenarios complejos y de constante vulnerabilidad. Actualmente el mundo enfrenta una crisis sanitaria ante la cual todos somos vulnerables; la medida más efectiva ante ésta es la distancia física y permanecer, en medida de lo posible, en casa. Dicha situación provoca la necesidad de analizar qué representa para estas mujeres experimentar este confinamiento

Palabras clave: COVID-19, desapariciones, vulnerabilidad, resiliencia

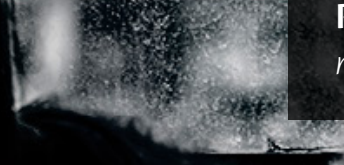

\title{
La vulnerabilidad
} de quienes han sido vulneradas. Mujeres con familiares desaparecidos frente al COVID-19

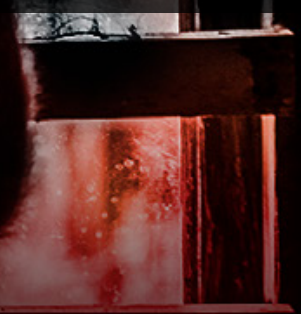
y los destellos de resiliencia.

Las mujeres con familiares desaparecidos en México, comenzaron el año 2020 con múltiples situaciones complejas y retos desafiantes. Recién terminaban las fiestas familiares decembrinas y, desde mi experiencia, puedo señalar que en muchos casos se comenzaban a generar procesos de restauración emocional, debido a que las emociones que se desarrollan en fechas como Navidad y año nuevo son acompañadas por el profundo dolor de la ausencia. En los primeros días de febrero se anunciaba por el gobierno federal la cifra de 61637 personas desaparecidas. Además, se reconoció la existencia de 3631 fosas clandestinas (Comisión Nacional de Búsqueda, 2020) y 30 mil cuerpos sin identificar en los servicios forenses (Comisión Nacional de Derechos Humanos, 2020).

En concordancia con estos datos, es importante puntualizar que existen grandes desafíos a encarar por las autoridades mexicanas, pues desde hace años no ha existido capacidad institucional

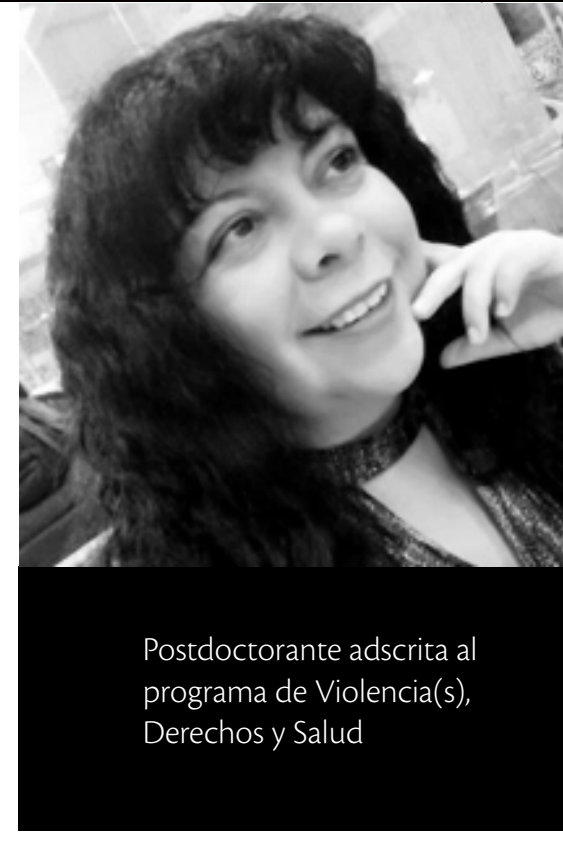



tente, son principalmente las mujeres quienes se mantienen en búsqueda constante; parte de ello se debe a los estrechos vínculos afectivos con quienes desaparecen. Dada esta condición, a principios de año diversos colectivos conformados principalmente por mujeres que buscan a sus familiares desaparecidos, se organizaban para iniciar brigadas de búsqueda en diferentes regiones del país; sin embargo, sus planes y actividades fueron interrumpidas debido a la crisis sanitaria provocada por la nueva cepa de coronavirus SARS-CoV-2, mejor conocida como covid-19.

\section{Una mirada a las múltiples implicaciones de la desaparición}

Dentro de las expresiones de violencia más graves se encuentra la desaparición forzada, debido a que existen sentimientos de incertidumbre que acompañan al sufrimiento de la familia, a causa de la existencia de una pérdida ambigua (Meza, García, Torres, Castillo, Suárez y Martínez, 2008). Perder a un ser querido de esta manera, provoca lo que Cabodevilla (2007) ha nombrado duelo ambiguo, en éste, las personas perciben a la persona ausente físicamente, pero presente psicológicamente (por lo regular son procesos largos y difíciles de cerrar). Además, la experiencia de vivir el duelo no siempre es posible frente a una desaparición forzada, padecer la desaparición de un ser querido conlleva a asumir la existencia de un profundo dolor, donde sentimientos de culpa y angustia se acentúan y evidencian de forma particular, pues la incógnita sobre el fin de su ser querido es una cuestión tormentosa (Delgado, 2014).

Para Bezanilla y Miranda (2014) en aquellas familias donde ha ocurrido una desaparición forzada de uno o más integrantes, se han advertido procesos de "duelos alterados", los cuales se caracterizan por la presencia constante de incertidumbre, en los que deriva el trauma; la familia queda "suspendida" en un momento, con la pregunta constante sobre el paradero de la persona ausente; se observan bloqueos en la capacidad del duelo y manejo de pérdidas, lo que genera una traumatización continua.

Las historias de familias y personas que tienen seres queridos desaparecidos, se acompañan de diferentes episodios de vulnerabilidad, violencia y vulneración. La proliferación de la violencia que se suscitó en México durante los últimos 12 años, las posicionó en un constante estado de vulnerabilidad. Hay que puntualizar que la desaparición forzada es un delito de carácter múltiple, continuo e imprescriptible; es decir, la desaparición forzada viola a su vez otros derechos humanos, ya que mientras no se sepa del paradero de la víctima se sigue perpetuando sin que haya obstáculos temporales para la acción punitiva (Cerezo, 2018). Además, Mercado (2017) argumenta que, cuando la justicia opera de manera efectiva, se convierte en un dispositivo para los procesos de duelo bajo contextos violentos; cuando no lo hace se vuelve un obstáculo, un factor de revictimización y contribuye a la perpetuación del dolor. Sin duda, la situación que viven las familias con integrantes desaparecidos provoca implicaciones terribles que son acompañadas por una constante angustia e incertidumbre.

Ante un panorama marcado por la desaparición, se podría pensar que las situaciones de adversidad que puede resistir una persona no podrían empeorar; sin embargo, el año 
2020 traía consigo un escenario mundial que posicionaría a toda la humanidad ante un estado de vulnerabilidad ineludible y que haría más complejo el mundo cotidiano. Los planes y proyectos elaborados a nivel global, entrarían en una completa transformación, o en el mejor de los casos en un estado de latencia, debido a la pandemia covid-19.

\section{Vulnerabilidad que se incrementa}

De acuerdo con Feito (2007,p. 11) existen "espacios de vulnerabilidad", los cuales refieren a condiciones desfavorables o centros de confluencia de amenazas potenciales, que en palabras del autor: "exponen a las personas a mayores riesgos, a situaciones de falta de poder o control, a la imposibilidad de cambiar sus circunstancias y, por lo tanto, a la desprotección". De esta manera, se puede visualizar que los "espacios de vulnerabilidad" facilitan la articulación de diversas condiciones que inciden en el grado de vulnerabilidad: exposición al riesgo, incapacidad de control sobre los eventos y la potencialidad a sufrir algún daño. Chardon (2002), desde una visión integral, propone examinar la vulnerabilidad como un sistema dinámico y complejo, que se genera por diversos factores asociados a procesos físico-naturales, socioeconómicos, político-institucionales, entre otros.

Pero ¿Qué representa para las mujeres con familiares desaparecidos enfrentar sus espacios de vulnerabilidad frente a la pandemia? De acuerdo con las investigaciones de corte cualitativo que he realizado, las mujeres que tienen familiares desaparecidos experimentan sentimientos de agobio y desesperación cuando por largos periodos se encuentran en casa. Entre sus argumentos ellas manifiestan que cuando no se desarrollan actividades relacionadas a la demanda de justicia, a la búsqueda de sus familiares, a la divulgación de fichas de búsqueda —entre otras actividades—, se genera en ellas un sentimiento de impotencia, desesperación y ansiedad; incluso su salud física también se ve afectada, la inactividad les incrementa dolores y diversos malestares; además, muchas de las mujeres que tienen familiares desaparecidos son adultas mayores, y padecen enfermedades crónicas degenerativas como diabetes e hipertensión, que en función con la información que he colectado por años, se desarrollan o se agudizan en el momento que sus familiares desaparecen.

Durante estos días he mantenido conversaciones con diversas mujeres que tiene familiares desaparecidos y he podido observar que, para algunas de ellas, el confinamiento ha significado una profunda desolación que ha incidido sobre su salud física, provoca una agudización en los sentimientos de incertidumbre y, en consecuencia, afecta su estado emocional. De acuerdo con sus experiencias, el paso del tiempo es devastador porque afecta las posibles evidencias físicas en las regiones e incide sobre la localización de testigos clave. Por ello, detener las búsquedas es un hecho trágico que les genera una fundamentada impotencia.

Los procesos de búsqueda tanto en vida, como de restos humanos - aun cuando se desarrollan en escenarios de verdadero horror y desolación-, son un aliciente que permite dar sentido a las vidas de quienes están buscando un ser querido. Dar nuevos sentidos al trauma que se ha vivido es un factor importante para generar procesos resilientes. Es decir, permite la reconstrucción psíquica y la elaboración de nuevos proyectos de vida. Se entiende que la resiliencia es un proceso en constante desarrollo que inicia después de una agonía psíquica (Cyrulnik, 2014). Al elegir sobreponerse, se abren opciones que facilitan al sujeto comprenderse dentro de su propia historia, y reconstruirse a través de actividades que le permitan dar significado a su herida; como lo son los procesos creativos, donde surge una interpretación de lo acontecido y se puede expresar en acciones solidarias hacia 
los pares o en actividades artísticas catárticas (Cyrulnick, 2001). En estos casos, muchos de los proyectos de vida se rigen bajo una base medular, que es buscar a quien está desaparecido.

\section{Destellos resilientes}

Frente a esta nueva realidad del covid-19, muchas mujeres dieron paso a acciones creativas y comenzaron a intensificar su actividad en redes sociales virtuales, para promover de manera más insistente fichas de búsqueda y mensajes de demanda con el objeto de promover conciencia social y política. Asimismo, diferentes colectivos se organizaron para realizar una marcha virtual el 10 de mayo, fecha de gran peso cultural y significativo para las madres que tienen hijos desaparecidos, desde plataformas virtuales como Facebook y Twitter se manifestó un acompañamiento a través de fotos y videos que mostraban mensajes de solidaridad, demanda de justicia y búsqueda. Algunas de las frases más pronunciadas fueron:

\section{¡No están solas! \\ Corazones en marcha \\ Hasta encontrarles}

Las madres de desaparecidos/as se acompañaron tanto por otras madres en la misma situación, como por personas solidarias, las cuales mostraron gran empatía para participar en una marcha virtual inédita, fruto de un confinamiento obligado. Los espacios construidos de forma colectiva (aun virtuales a través de Facebook y Twitter) significan un aliciente para sentirse acompañadas, lo cual les da oportunidad para generar recursos

ca

la resiliencia puede promoverse

a nivel grupal, comunitario,

social y político."

que permiten afrontar el dolor, organizarse y producir acciones que complementan sus nuevos objetivos y proyectos de vida. Cabe mencionar que la ausencia o la presencia de apoyo social, constituye un factor importante para la elaboración de procesos resilientes. De igual forma, las condiciones de precariedad o los escenarios de riesgo por la violencia persistente, pueden ser enfrentados de manera más efectiva cuando se cuenta con redes de apoyo a nivel emocional y social, tanto fuera como dentro de la familia (Díaz, Molina y Marín, 2015). Lo anterior remite a Vergely (2003, p. 56) quien sostiene que:

Cuando uno está sometido a la conmoción de una desgracia, se comienza a salir de la obsesión de los pensamientos obsesivos gracias a la mediación de los otros. Y es que, gracias a que el otro viene a sostener a aquel o a aquella que ya no puede sostenerse, podrá, estando así sostenido, aquel o aquella que no se sostiene, reafirmar el pie. Es un gesto profundo que viene a borrar el gesto de la violación y de la transgresión.

Dadas estas acciones solidarias, tanto en tiempos de pandemia como antes de ésta, que inciden sobre los procesos de resiliencia de estas mujeres, coincido con Cyrulnik (2000), cuando comenta que uno puede descubrir en sí mismo y en el ambiente que lo rodea algunos medios para volver a la vida y retomar el camino del desarrollo, conservando al mismo 
tiempo en la memoria el recuerdo de la herida. Sin embargo, debemos considerar que la resiliencia no es absoluta y tampoco se adquiere para siempre, que responde a un proceso dinámico, al contexto, al desarrollo del trauma y a los recursos personales, familiares y sociales con los que se cuentan (Torralba, 2013). De ahí la importancia e insistencia de visibilizar la vulnerabilidad de quienes han sido vulneradas por la desaparición de un ser querido y de promover las alternativas para acompañarlas.

\section{Destellos que pueden convertirse en una luz intermitente}

Ante una vulnerabilidad generalizada conviene preguntarse ¿Es posible para las instituciones gubernamentales responder a la demanda de atención y búsqueda en tiempos de pandemia? La respuesta es que sí es posible. Y esta fue manifestada por diversos colectivos de personas que buscan desaparecidos a través de sus redes sociales. Los argumentos principales para sustentar dicha afirmación fueron que podían realizar desde el confinamiento las siguientes actividades:

- Actualización de expedientes, sobre todo aquellos donde se presentaban inconsistencias.

- Entrevistas virtuales a testigos potenciales a través de plataformas de internet, en calidad de testimonio.

- Atender las fallas procesales en la documentación y registro de información.

- Revisar, incrementar y mejorar las bases de datos.

- Profundizar de manera documental sobre las formas criminales en que ocurrió la desaparición y registrar similitudes conforme a la región y el año.

Frente a un fenómeno tan doloroso que caracteriza la historia contemporánea de nuestro país, es preciso demostrar más que voluntad política para atender el problema, se requieren de acciones en conjunto que respondan a una estructura comprometida y eficiente. Si bien las mujeres que buscan a sus desaparecidos, han demostrado con sus acciones no asumir un papel pasivo de víctimas, generando capacidades gestoras y desarrollando procesos resilientes para sobrellevar la adversidad. No se les debe asignar un papel de "heroínas", capaces de resistir (sin procurar el autocuidado) y desempeñar acciones - por demás desgastantes-, ante una evidente falta de eficacia de las respuestas institucionales.

\section{Reflexiones para seguir insistiendo}

La respuesta resiliente frente a la adversidad que la pandemia provoca, no es exclusiva de los individuos, la resiliencia puede promoverse a nivel grupal, comunitario, social y político. Dentro de los pilares para promover la resiliencia se encuentra la flexibilidad y la capacidad creativa (Quiñonez, 2007), dichos pilares pueden promoverse y fortalecerse en plena adversidad sanitaria. La resiliencia es una tarea que desarrollar también por las propias

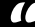

la oportunidad perfecta

para acompañar y generar comunidad, instituciones gubernamentales, por las fiscalías asignadas para la búsqueda, y por los servidores públicos; de esta forma, pueden sobrellevar también sus propias adversidades y dar respuestas más eficaces. El hecho de que la resiliencia sea una alternativa 
relacionada al empoderamiento, esta no exime a los gobiernos de procurar la generación de bienestar de sus ciudadanos (Evans y Reid, 2015). Se entiende que las implicaciones de una violencia extrema, como las que proliferó de manera alarmante en poco más de 12 años, no pueden resolverse en poco tiempo y tampoco están libres de complejidades; pero, sí existen mecanismos que pueden implementarse bajo constantes análisis de efectividad y en conjunto con quienes tienen más experiencia: las personas que buscan a sus desaparecidos.

La crisis sanitaria debe observarse como una oportunidad para reflexionar sobre situaciones de vulnerabilidad más graves, las cuales pueden mitigarse fomentando una cultura de solidaridad, empatía, fraternidad y compasión. No existen recetas para generar resiliencia; el proceso es diferenciado en cada individuo, grupo, comunidad y sociedad, pero existen diversos caminos y recursos para construirla (Torralba, 2013). La actitud que tomemos frente a los escenarios adversos y la promoción de una cultura resiliente serán pilares clave para sobrellevar la adversidad y transformar las formas de relacionarnos, pues la resiliencia no puede ocurrir a costa del sufrimiento de otros o de la indiferencia asumida frente a sus problemas. La resiliencia es algo que se construye colectivamente, algo que se humaniza y que da lugar a cada uno, que funciona como una palanca terapéutica que permite experiencias de resiliencia colectiva, en donde se crean nuevas formas relacionales constructivas y evolutivas (Hendrick y Denis, 2018). Por ello, me atrevo a recomendar, el no dejar de mirar a quienes enfrentan problemas más complejos, como es el caso de las familias de personas desaparecidas. Los tiempos del covid-19, son la oportunidad perfecta para acompañar y generar comunidad, una comunidad resiliente.

\section{Referencias bibliográficas}

Bezanilla, J. M. y Miranda, A.(2014). Violaciones graves a derechos humanos y su impacto familiar. Revista Cuadernos de Crisis, 2 (15), 1-9.

Cabodevilla, I. (2007). Las pérdidas y sus duelos. Revista Anales del sistema sanitario de Navarra, 3 (30), 163-176.

Cerezo, H. (2018). Vivos los queremos. Claves para entender la desaparición forzada en México. Viandante.

Chardon, A. C. (2002). Un enfoque geográfico de la vulnerabilidad en zonas urbanas expuestas a amenazas naturales: El ejemplo andino de Manizales, Colombia. Universidad Nacional de Colombia, Sede Manizales.

Cyrulnik, B. (2014). La resiliencia en el siglo xxi. En: Madariaga, J. M. (Coord.), Nuevas miradas sobre la resiliencia. Ampliando ámbitos y prácticas (pp. 31-51). Gedisa.

Cyrulnik, B. y Bixio, A. (2007). De cuerpo y alma: Neuronas y afectos: la conquista del bienestar. Gedisa.

Cyrulnik, B. (2001). La Maravilla del dolor: El sentido de la resiliencia. Granica.

Delgado, R. (2014). Elaboración del duelo de una madre cuyo hijo trabajaba como sicario en Ciudad Juárez, Chihuahua. Nóesis. Revista de Ciencias Sociales y Humanidades, 23 (46), 224-244.

Díaz, V. E., Molina, A. y Marín, M. (2015). Las pérdidas y los duelos en personas afectadas por el desplazamiento forzado. Revista Pensamiento Psicológico, 13 (1). 65-80.

Evans, B., Reid, J. y Altamirano, V. (2016). Una vida en resiliencia: El arte de vivir en peligro. Fondo de Cultura Económica. 
Feito, L. (2007). Vulnerabilidad. En: Anales del sistema sanitario de Navarra. Madrid: Gobierno de Navarra-Departamento de Salud, 30 (3), 07-22.

Hendrick, S. y J. Denis (2018). Familias, psicosis, institución y corresiliencia. En: Cyrulnik, B. y Anaut, M. (Coords), Resiliencia y adaptación. La familia y la escuela como tutores de resiliencia. Gedisa.

Mercado Lenis, D. F. (2017). El duelo en contextos de violencia. Aportes desde el psicoanálisis y la perspectiva transcultural. Revista Lumen Gentium, 1 (1), 35-43.

Dávalos, E. G. M., García, S., Gómez, A. T., Castillo, L., Suárez, S. S. y Silva, B. M. (2008) . El proceso del duelo. Un mecanismo humano para el manejo de las pérdidas emocionales. Revista de Especialidades Médico-Quirúrgicas, (13)1, 28-31.

Quiñones, M. A. (2007). Resiliencia: Resignificación creativa de la adversidad. Universidad Distrital Francisco José de Caldas.

Martín Torralba, L. (2013). La personalidad resiliente. Síntesis.

Vergely, B. (2003) Enfoque filosófico de la resiliencia. En: Cyrulnik, B., Tomkiewicz, S., Guénard, T., Vanistendael, S., Manciaux M. y otros. El realismo de la esperanza. Testimonios de experiencias profesionales en torno a la resiliencia (pp. 51-67). Gedisa. 